\title{
A PARABOLIC REGULARIZATION PROPERTY OF $p$-LOGARITHMIC SOBOLEV GENERATORS
}

\author{
GABRIELE GRILLO
}

Abstract. Let $N$ be a Riemannian manifold, $M \subset N$ be a domain with smooth boundary, $\mu$ a positive measure on $M$ such that $M$ has unit $\mu$-volume. Consider the evolution driven by the $p$-Laplace-type operator $(p>2)$ associated to the natural $p$-energy functional $\mathscr{E}(p)$ constructed from $\mu$, homogeneous Dirichlet boundary conditions on $\partial M$ being assumed. Assume that a single suitable logarithmic inequality holds for $\mathscr{E}^{(p)}$.Then we show that the evolution brings any data belonging to the natural domain of the evolution instantaneously into $\mathrm{L}^{q}$ for any $q>2$, with quantitative bounds on the $\mathrm{L}^{q}$ norms.

\section{Mathematics subject classification (2000): 47H20, 35K55, 58J35.}

Keywords and phrases: $p$-Laplacian, nonlinear evolutions on manifolds, logarithmic Sobolev inequality, contractivity properties.

\section{REFERENCES}

[1] C. Ané, S. Blachère, D. Chafaï, P. Fougères, I. Gentil, F. Malrieu, C. Roberto, G. Scheffer, Sur les inégalités de Sobolev logarithmiques, Panoramas et Synthèses 10. Société Mathématique de France, Paris, 2000.

[2] N. Alikakos, R. Rostamian, Lower bound estimates and separable solutions for homogeneous equations of evolution in Banach space, J. Differential Equations, 43 (1982), 323-344.

[3] M. Biroli, U. Mosco, Sobolev inequalities on homogeneous spaces, Potential Anal., 4 (1995), 311-324.

[4] H. BrezIS, Operateurs Maximaux Monotones', North-Holland Publishing Company, Amsterdam/London, 1973.

[5] F. Cipriani, Sobolev-Orlicz embeddings, weak compactness, and spectrum, J. Funct. Anal., 177 (2000), 89-106.

[6] F. CIPRIANI, G. GRILlo, Uniform bounds for solutions to quasilinear evolution equations, J. Differential Equations, 177 (2001), 209-234.

[7] F. Cipriani, G. GRILlo, Nonlinear Dirichlet forms and nonlinear Markov semigroups, J. Reine Angew. Math., 562 (2003), 201-235.

[8] E. DiBenedetto, Degenerate Parabolic Equations, Springer Verlag, New York/Berlin, 1993.

[9] P. Federbush, A partially alternate derivation of a result of Nelson, J. Math. Phys., 10 (1969), $50-52$.

[10] B. Franchi, C. Perez, R.L. Wheeden, Self-improving properties of John-Nirenberg and Poincaré inequalities on spaces of homogeneous type, J. Funct. Anal., 153 (1998), 108-146.

[11] L. Gross, Logarithmic Sobolev inequalities, Amer. J. Math., 97 (1976), 1061-1083.

[12] L. Gross, Logarithmic Sobolev inequalities and contractivity properties of semigroups, in: Dirichlet Forms, Lecture Notes in Mathematics 1563, pp. 54-88.

[13] E. Nelson, The free Markoff field, J. Funct. Anal., 12 (1973), 211-227. 
[14] R.E. Showalter, Monotone Operators in Banach Space and Nonlinear Partial Differential Equations', Mathematical Surveys and Monographs, vol. 49, American Mathematical Society, 1997.

[15] B. SIMON, R. HøEGH-KROHN, Hypercontractive semigroups and two dimensional self-coupled Bose fields, J. Funct. Anal., 9 (1972), 121-180. 